\title{
Physical Activity Levels in Peripheral Artery Disease Patients
}

\author{
Aline Mendes Gerage, ${ }^{1}$ Marilia de Almeida Correia, ${ }^{2}$ Paulo Mesquita Longano de Oliveira, ${ }^{2}$ Aline Cabral Palmeira, ${ }^{3}$ \\ Wagner Jorge Ribeiro Domingues, ${ }^{4}$ Antônio Eduardo Zeratti, ${ }^{5}$ Pedro Puech-Leão, ${ }^{5}$ Nelson Wolosker, ${ }^{3}$ Raphael \\ Mendes Ritti-Dias, ${ }^{2}$ Gabriel Grizzo Cucato ${ }^{3}$ \\ Universidade Federal de Santa Catarina - Departamento de Educação Física, ${ }^{1}$ Florianópolis, SC - Brazil \\ Universidade Nove de Julho, ${ }^{2}$ São Paulo, SP - Brazil \\ Hospital Israelita Albert Einstein, ${ }^{3}$ São Paulo, SP - Brazil \\ Universidade Federal do Amazonas, ${ }^{4}$ Parintins, AM - Brazil \\ Universidade de São Paulo Faculdade de Medicina Hospital das Clinicas, ${ }^{5}$ São Paulo, SP - Brazil
}

\begin{abstract}
Background: Increases in daily physical activity levels is recommended for patients with peripheral artery disease (PAD). However, despite this recommendation, little is known about the physical activity patterns of PAD patients.

Objective: To describe the physical activity patterns of patients with symptomatic peripheral artery (PAD) disease.

Methods: This cross-sectional study included 174 PAD patients with intermittent claudication symptoms. Patients were submitted to clinical, hemodynamic and functional evaluations. Physical activity was objectively measured by an accelerometer, and the time spent in sedentary, low-light, high-light and moderate-vigorous physical activities (MVPA) were obtained. Descriptive analysis was performed to summarize patient data and binary logistic regression was used to test the crude and adjusted associations between adherence to physical activity recommendation and sociodemographic and clinical factors. For all the statistical analyses, significance was accepted at $\mathbf{p}<0.05$.
\end{abstract}

Results: Patients spent in average of $640 \pm 121 \mathrm{~min} /$ day, $269 \pm 94 \mathrm{~min} / \mathrm{day}, 36 \pm 27 \mathrm{~min} / \mathrm{day}$ and $15 \pm 16 \mathrm{~min} / \mathrm{day}$ in sedentary, low-light, high-light and MVPA, respectively. The prevalence of patients who achieved physical activity recommendations was $3.4 \%$. After adjustment for confounders, a significant inverse association was observed between adherence to physical activity recommendation and age $(O R=0.925 ; p=0.004)$, while time of disease, ankle brachial index and total walking distance were not associated with this adherence criteria $(p>0.05)$.

Conclusion: The patterns of physical activity of PAD patients are characterized by a large amount of time spent in sedentary behaviors and a low engagement in MVPA. Younger patients, regardless of the clinical and functional factors, were more likely to meet the current physical activity recommendations. (Arq Bras Cardiol. 2019; 113(3):410-416)

Keywords: Motor Activity; Exercise; Waling; Peripheral Arterial Disease; Intermittent Claudication.

\section{Introduction}

Patients with peripheral artery disease (PAD) and symptoms of intermittent claudication have walking impairment, several comorbid conditions and increased cardiovascular risk, ${ }^{1,2}$ due to the disease characteristics and severity. Supervised exercise training has been considered a cornerstone in the clinical therapeutic approach in PAD patients, ${ }^{3}$ as it improves several components of physical function and quality of life..$^{4-6}$ Similarly, positive effects of device-monitored, home-based exercise training programs to improve the walking capacity in these patients have also been reported. ${ }^{7}$ However,

Mailing Address: Raphael Mendes Ritti-Dias •

Universidade Nove de Julho - Programa de Pós-Graduação em Ciências da Reabilitação - Rua Vergueiro, 235. Postal Code 01504-000, São Paulo, SP - Brazil

E-mail: raphaelritti@gmail.com

Manuscript received October 16, 2018, revised manuscript December 09, 2018, accepted January 16, 2019

DOI: $10.5935 / \mathrm{abc} .20190142$ these interventions are available for a restricted number of patients, limiting applicability in the public health context. Therefore, recommendations to increase physical activity levels remain the most often used approach in clinical practice.

Current physical activity recommendations for the overall population, including PAD patients, consists of practicing at least $150 \mathrm{~min}$ of moderate or $75 \mathrm{~min}$ of vigorous physical activities or an equivalent combination of moderate-vigorous physical activities (MVPA) per week. ${ }^{8}$ Furthermore, it has been recommended that MVPA should be performed in bouts with at least a 10-minute duration. ${ }^{8}$ Surprisingly, there are no data indicating the number of symptomatic PAD patients who achieve these physical activity recommendations. Given that most of symptomatic PAD patients are older, have several comorbidities, and that symptoms of intermittent claudication are the main barrier for physical activity practice in these patients, ${ }^{9}$ by limiting their walking and functional capacity, it is expected that only a small percentage of the patients would achieve the recommended physical activity levels.

Thus, in this study we aimed to describe the physical activity pattern of Brazilian patients with PAD and symptoms 
of intermittent claudication according to the recommendations for physical activity practice, providing objective information regarding the time spent in sedentary behavior, light physical activity and MVPA. Moreover, we tested the association between adherence to physical activity recommendations and sociodemographic and clinical factors in Brazilian patients with symptomatic PAD.

\section{Methods}

\section{Study design and ethical issues}

This descriptive study was approved by Local Ethics Committee. Prior to data collection, patients were informed about the methodological and logistic procedures required to participate in the study, as well as the risks and benefits, and signed a written informed consent form before participation.

\section{Participants}

The overall sample consisted of symptomatic PAD patients, recruited at a tertiary center specialized in vascular disease, between September 2015 and November 2017. The tertiary center is a specific unit designed to treat PAD patients with intermittent claudication symptoms. There, physicians instruct patients to: stop smoking, control their risk factors, and increase their physical activity levels. In the present study, no additional instructions were given, and patients were asked to keep their physical activity routine. To be included in the present study, patients should: have PAD (Fontaine Stage II), ankle brachial index $(\mathrm{ABI})<0.90$ in one or both legs and undergo the six-minute walking test (6MWT). Patients with non-compressible vessels, amputated limbs and/or ulcers, previous diagnosis of neurological or psychiatric disorders, or those classified as illiterate were excluded.

\section{Measurements}

\section{Clinical data}

A standardized face-to-face interview was performed, including assessment of social and demographic information, co-morbid conditions (self-reported), and medications. Social and demographic variables included age and gender (male or female). Time of disease diagnosis was obtained through the question "How long have you had the disease?". Data on smoking habits (ex- or current smoker, or non-smoker), obesity (body mass index (BMI) $\geq 30 \mathrm{~kg} / \mathrm{m}^{2}$ ), diabetes (doctor-diagnosed or hypoglycemic drugs), hypertension (systolic/diastolic blood pressure $>140 / 90 \mathrm{mmHg}$ or antihypertensive drug use), dyslipidemia (doctor-diagnosed or hypolipidemic drug use), coronary heart disease, heart failure and history of cancer (self-reported or analysis of medical records) were obtained.

\section{Disease severity}

PAD severity was obtained by calculating the $A B I$ in accordance with the guidelines. ${ }^{10}$ All measures were carried out by a single and trained evaluator, using vascular Doppler (Medmega DV160, Brazil) and aneroid sphygmomanometer.

\section{Walking capacity}

The 6MWT was performed on a 30-meter long corridor, following the previously described protocol. ${ }^{11}$ Briefly, patients were instructed to complete as many laps as possible. Patients were encouraged to "walk at the usual pace for six-minutes and cover as much ground as possible". Patients were informed that they could rest, if necessary. At the end of each minute, patients received feedback on the elapsed time and standardized encouragement in the form of statements such as "you are doing well, keep it up" and "do your best". Total walking distance was defined as the maximum distance which the patient could walk during the test, with or without leg pain. In addition, the self-reported ambulatory ability was assessed using the Brazilian versions of Walking Impairment Questionnaire $(\mathrm{WIQ})^{12}$ and the Walking Estimated-Limitation Calculated by History (WELCH) questionnaire. ${ }^{13}$

\section{Objectively measured physical activity}

Physical activity was assessed using a GT3X + triaxial accelerometer (Actigraph, Pensacola, FL, USA). Each participant was instructed to use the accelerometer for seven consecutive days, removing it only for sleeping, bathing or performing activities in the water. The device was attached to an elastic belt and attached to the right side of the hip. Data reduction was performed using the Actilife software, version 6.02 (Actigraph, Pensacola, FL, USA), with a $60 \mathrm{~Hz}$ sample frequency and 60 s epochs. Periods with consecutive values of zero for $60 \mathrm{~min}$ or longer were interpreted as "accelerometer not worn" and excluded from the analysis. Physical activity data were included only if the participant had accumulated a minimum of 10 hours/day of recording for at least four days, including one weekend day. The average of total time spent in each intensity of physical activity was calculated using the cutoff points specific for elderly individuals, ${ }^{14}$ adapted by Buman et al., ${ }^{15}$ considering sedentary time (SED) as 0 - 99 counts/min; low-light physical activities as 100-1040 counts/min, high-light physical activities as 1041-1951 counts/min and MVPA as $\geq 1952$ counts/min using the vertical axis, and analyzed in min/day, adjusting for the time and number of days the device was worn. The total time spent in SED bouts and the time spent in bouts of at least high-light physical activities and MVPA were analyzed by the sum of minutes spent in SED, high-light physical activities and MVPA, respectively, in periods lasting $\geq 10$ minutes. Additionally, we calculated the percentage of patients that met the current physical activity recommendations ( $\geq 150 \mathrm{~min} /$ week) considering MVPA bouts.

\section{Statistical analysis}

The sample size was calculated by estimating an effect size of 0.3 in the chi-square analysis, considering an alpha error of $5 \%$ and a power of $80 \%$. The sample size required for the study was 143 participants. The data were stored and analyzed using the Statistical Package for the Social Sciences (SPSS, version 17.0, SPSS Inc, Chicago, IL). Descriptive analysis was performed to summarize the patients' data using means, standard deviation or frequency distribution (absolute and relative), as appropriate. Binary logistic regression was used to test the crude and adjusted (age, time of the disease 
diagnosis, ankle-brachial index, and six-minute walking distance) association between adherence to physical activity recommendation and sociodemographic data and clinical factors. The results are expressed as odds ratios (OR) and their respective $95 \%$ confidence intervals $(95 \% \mathrm{Cl})$. For all the statistical analyses, significance was set at $p<0.05$.

\section{Results}

The overall characteristics of patients are shown in Table 1. The mean age of all patients was $66.7 \pm 9.0$ years and, on average, patients had moderate disease (ABI: $0.61 \pm 0.18$ ). Most patients had hypertension (88.9\%), dyslipidemia (85.2\%) and diabetes (52.4\%), and used antihypertensive (78\%) (i.e. thiazide diuretics, calcium channel blockers, angiotensin-converting enzyme inhibitors, angiotensin II receptor antagonists, betablockers), lipid-lowering (89\%) (i.e. statins) and antiplatelet agent drugs (85\%) (i.e. irreversible cyclooxygenase inhibitors, adenosine diphosphate receptor inhibitors). Forty-three percent of the patients used antidiabetic (i.e. sulfonylureas, metformin, thiazolidinediones, alpha-glucosidase inhibitors, meglitinides), $29 \%$ used vasodilator (i.e. hydralazine and minoxidil) and $20 \%$ used antidepressant drugs (i.e. sertraline, fluoxetine, citalopram, escitalopram, paroxetine)

Figure 1 depicts the distribution of time spent in sedentary, low-light, high-light and moderate/vigorous activities. Patients, aged between 43 and 96 years, spent in average $640 \pm 121 \mathrm{~min} /$ day, $269 \pm 94 \mathrm{~min} /$ day, $36 \pm 27 \mathrm{~min} /$ day and $15 \pm 16 \mathrm{~min} /$ day in sedentary, low-light, high-light and moderate/vigorous physical activities, respectively. Most patients (52.9\%) spent less than $10 \mathrm{~min}$ in moderate/vigorous physical activities (sporadic, non-bouted) per day.

Table 2 depicts data about sedentary bouts ( $<100$ counts), bouts of high light and MVPA ( $\geq 1041$ counts) and bouts only of MVPA ( $\geq 1952$ counts). Ninety percent of patients spent at least 10 bouts in sedentary behavior per day and, on average, the total duration of this bout was $413.7 \pm 151.1 \mathrm{~min} /$ day. On the other hand, sedentary breaks lasted $174.4 \pm 51.4 \mathrm{~min} /$ day. Thirty-one percent of patients did not accumulate 10 or more consecutive minutes a week, at least, in high-light physical activities. Considering only MVPA, $67.7 \%$ of patients did not accumulate 10 consecutive minutes (bouts) or more at this intensity of physical activity during a week. Among those patients who spent at least one bout of MVPA, the duration of this bout was $9.7 \pm 9.6 \mathrm{~min} /$ day.

The prevalence of patients who achieved physical activity recommendations for the overall population ( $\geq 150 \mathrm{~min} /$ week of MVPA in bouts of 10 minutes or more) was only $3.4 \%$. Stratifying by age (Figure 2), this prevalence was $11.1 \%$ in those under 60 years old, $2.9 \%$ in those between 60 and 64 years old, and $1 \%$ in those over 65 years. No patients over 70 years old achieved the physical activity recommendations for the overall population.

Table 3 shows crude and adjusted association between adherence to physical activity recommendations and sociodemographic and clinical characteristics in PAD patients. After adjustment for confounders, an inverse and significant association was observed between adherence to physical activity recommendation and age $(\mathrm{OR}=0.867 ; \mathrm{p}=0.011)$,
Table 1 - Characteristics of peripheral artery disease patients according to gender $(n=174)$

\begin{tabular}{|c|c|}
\hline & Values \\
\hline Age (years) & $66.7(9.0)$ \\
\hline Gender ( $\%$ men) & 61.5 \\
\hline Still working (\%) & 20.5 \\
\hline Time of disease diagnosis (yrs.) & $7.9(5.8)$ \\
\hline Ankle-brachial index & $0.61(0.18)$ \\
\hline Claudication distance $(\mathrm{m})^{\dagger}$ & $135.9(82.4)$ \\
\hline Six-minute walking distance (m) & $326.6(92.7)$ \\
\hline WIQ distance (score) & $22.7(22.2)$ \\
\hline WIQ speed (score) & $23.2(15.6)$ \\
\hline WIQ stairs (score) & $30.7(25.3)$ \\
\hline WELCH (score) & $27.3(19.1)$ \\
\hline \multicolumn{2}{|l|}{ Comorbidities and risk factors } \\
\hline Charlson index (score) & $3.0(1.7)$ \\
\hline Current smokers (\%) & 18.1 \\
\hline Hypertension (\%) & 88.9 \\
\hline Dyslipidemia (\%) & 85.2 \\
\hline Diabetes (\%) & 52.4 \\
\hline Obesity (\%) & 28.6 \\
\hline Coronary artery disease (\%) & 34.5 \\
\hline Heart failure (\%) & 13.6 \\
\hline Cancer (\%) & 14.9 \\
\hline \multicolumn{2}{|l|}{ Medications } \\
\hline Antihypertensive (\%) & 78 \\
\hline Antidiabetic (\%) & 43 \\
\hline Vasodilator (\%) & 29 \\
\hline Lipid-lowering (\%) & 89 \\
\hline Antiplatelet agent (\%) & 85 \\
\hline Antidepressants (\%) & 20 \\
\hline \multicolumn{2}{|l|}{ Medications } \\
\hline Cardiac (\%) & 24 \\
\hline Vascular (\%) & 12 \\
\hline
\end{tabular}

WIQ: Walking Impairment Questionnaire; WELCH: Walking EstimatedLimitation Calculated by History.

which means that for each year of life, the odds are $\sim 13 \%$ less to meet the physical activity recommendations. Time of disease diagnosis, $\mathrm{ABI}$ and total walking distance were not associated with this adherence criterion $(p>0.05)$.

\section{Discussion}

The main findings of the present study were: a) Brazilian PAD patients with intermittent claudication symptoms spent most part of the day in sedentary behaviors with a short time in MVPA; b) only $3.4 \%$ of the patients met the physical activity 


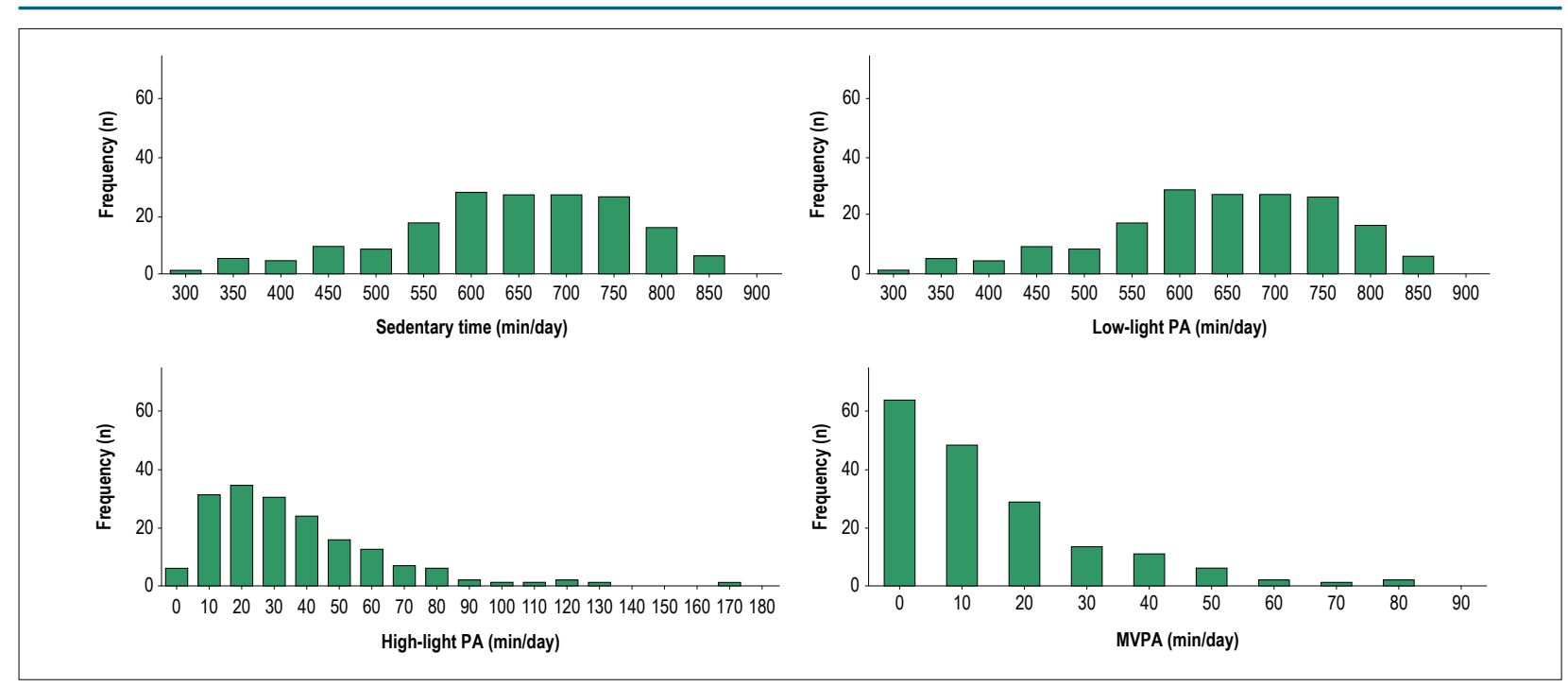

Figure 1 - Time spent in sedentary, low light, high light and moderate-to-vigorous (MVPA) physical activities (PA).

Table 2 - Total time spent in sedentary, high light or MVPA and MVPA bouts and sedentary breaks per week and per day in PAD patients $(n=174$ )

\begin{tabular}{lcc}
\hline Variable & In a week (mean \pm SD) & In a day (mean \pm SD) \\
\hline Total SED bouts & $120.1 \pm 32.6$ & $17.2 \pm 4.7$ \\
Total time in SED bouts $(\mathrm{min})$ & $2895.6 \pm 1057.3$ & $413.7 \pm 151.1$ \\
Total SED breaks & $118.7 \pm 32.6$ & $17.0 \pm 4.7$ \\
Total time in SED breaks $(\mathrm{min})$ & $8543.9 \pm 2518.0$ & $174.4 \pm 51.4$ \\
Total high light and MVPA bouts & $5.7 \pm 7.8$ & $0.8 \pm 1.1$ \\
Total time in high light and MVPA bouts $(\mathrm{min})$ & $84.01 \pm 123.8$ & $12.1 \pm 17.7$ \\
Total MVPA bouts & $1.5 \pm 3.1$ & $0.22 \pm 0.44$ \\
Total time in MVPA $(\mathrm{min})$ & $22.7 \pm 50.3$ & $3.2 \pm 7.2$ \\
\hline
\end{tabular}

SED: sedentary; MVPA: moderate/vigorous physical activity.

recommendations for the overall population; c) younger patients, regardless of clinical or physical factors, were more likely to meet the current physical activity recommendations for the overall population.

The cutoff used in the present study considered, in addition to "sedentary" and "moderate-to-vigorous physical activity", the "low-light" and "high-light" categories. ${ }^{15}$ This decision was based on the following aspects: a) light physical activities are the physical activities most often performed by the elderly, especially those with functional capacity limitations (i.e. patients with PAD); b) light physical activity was broadly unspecified to account for all activity between sedentary and moderate-to-vigorous physical activity (100-1,951 counts/minute); c) the association between light physical activity and health parameters increases when those light physical activities with high energy expenditure (high-light physical activity), that are closer to the classification of moderate-to-vigorous physical activities than sedentary activities, are considered. ${ }^{15}$

In the present study, our sample of PAD patients with intermittent claudication symptoms spent $640 \mathrm{~min} /$ day and
$15 \mathrm{~min} /$ day in sedentary behavior and MVPA, respectively, which represents $66.7 \%$ and $1.5 \%$ of the waking hours of the day. This pattern is similar to that observed in patients with other cardiovascular diseases, including coronary heart disease, congestive heart failure, myocardial infarction ${ }^{16}$ and stroke survivors. ${ }^{17}$ In these populations, sedentary behavior ranged from $576 \mathrm{~min} /$ day $^{16}$ to $606 \mathrm{~min} /$ day, $^{16,17}$ while MVPA ranged from $8.6 \mathrm{~min} /$ day to $11.4 \mathrm{~min} /$ day. Interestingly, although pain symptoms (intermittent claudication) during exercise have been reported as a main barrier for physical activity practice in PAD patients, ${ }^{9}$ their physical activity patterns seem to be similar to cardiac patients without walking impairment. The current physical activity recommendation for the overall population includes $150 \mathrm{~min} /$ day of MVPA in bouts of at least $10 \mathrm{~min}$. The results of this study indicated that a very small percentage $(3.4 \%)$ of our sample met the current physical activity recommendations. These values are lower than those of previous studies generally carried out with adults $(\sim 10 \%),{ }^{18}$ older adults $(12 \%)^{19}$ and osteoarthritis patients (13\% men and $8 \%$ women $)^{20}$ who usually also have physical limitations. The reduced number of patients who met the physical activity recommendations could be explained by the difficulty of 


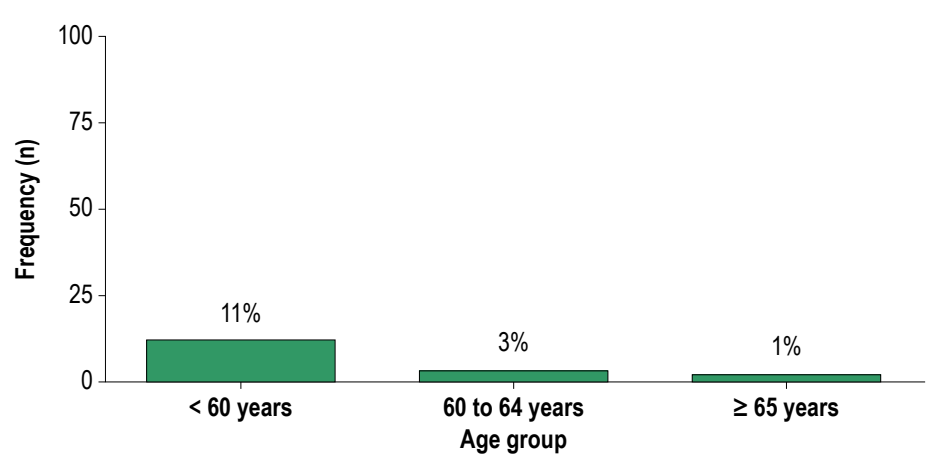

Figure 2 - Frequency of PAD patients who achieved the current physical activity recommendations according to age group.

Table 3 - Crude and adjusted association between adherence to physical activity recommendations and sociodemographic or clinical characteristics in PAD patients $(n=174)$

\begin{tabular}{lccc}
\hline \multirow{2}{*}{ Variable } & \multicolumn{2}{c}{ Crude analysis } & Adjusted analysis* \\
\cline { 2 - 4 } & OR $(95 \% \mathrm{Cl})$ & $\mathbf{p}$ & $\mathrm{OR}(95 \% \mathrm{Cl})$ \\
\hline Age & $0.87(0.79 ; 0.97)$ & 0.01 & $0.88(0.80 ; 0.98)$ \\
Time of disease diagnosis & $0.94(0.78 ; 1.12)$ & 0.46 & $0.98(0.83 ; 1.16)$ \\
Ankle-brachial index & $0.19(0.02 ; 153.41)$ & 0.77 & $1.14(0.07 ; 173.68)$ \\
Six-minute walking distance & $1.01(0.99 ; 1.02)$ & 0.12 & $1.00(0.99 ; 1.02)$ \\
\hline
\end{tabular}

*Adjusted by age, time of the disease diagnosis, ankle brachial index, and six-minute walking distance.

PAD patients to perform moderate and/or vigorous physical activities. In fact, as higher-intensity physical activities may precipitate the occurrence of intermittent claudication symptoms, PAD patients commonly perform lower-intensity physical activities to avoid the symptoms.

In the present study, we also analyzed the frequency of patients who achieved the current physical activity recommendations according to age group. We observed that no patients over 70 years old met the current physical activity recommendations for the overall population. This result was confirmed by the multivariate analysis, which revealed that younger patients are more likely to achieve the current physical activity recommendations. These results are in accordance with previous studies carried out with a representative sample of adults from the United States ${ }^{21}$ and with older adults in a population-based sample from Brazil, ${ }^{19}$ which showed an inverse relationship between age and the amount of time spent in MVPA physical activities. The decrease in physical activity with increasing age might be due to a worsening in physical functions associated with the presence of the comorbid conditions, leading to an increase in sedentary behavior and functional capacity impairment.

The $\mathrm{ABI}$, considered one of the best prognostic indexes in $\mathrm{PAD},{ }^{22}$ and walking capacity, a main clinical marker of PAD associated with endothelial function ${ }^{23}$ inflammation ${ }^{24}$ and several clinical indicators, ${ }^{2,25}$ were not associated with the meeting of physical activity recommendations. These results are not surprising, since $\mathrm{ABI}^{26}$ and walking capacity have been poorly associated with physical activity in patients with PAD. ${ }^{27}$
Previous studies showed that low levels of physical activity and high levels of sedentary behavior were associated with several risk factors, such as high blood pressure, ${ }^{28}$ increased arterial stiffness, ${ }^{29}$ increased waist circumference and reduced HDL cholesterol, ${ }^{30,31}$ in healthy and clinical populations. In symptomatic PAD patients, a study carried out by Garg et al. ${ }^{32}$ reported that reduced physical activity was associated with increased mortality and cardiovascular events. In other words, patients who attempted to control or eliminate their intermittent claudication symptoms by reducing their physical activity, worsened their risk of myocardial infarction, stroke, and death. Thus, the finding of our study that the majority of PAD patients did not attain the current physical activity recommendations highlights the necessity of interventions to increase physical activity in these patients. Future studies are necessary to describe whether different forms of exercise, home-based programs or wearable physical activity monitors are more effective to help patients to attain the current physical activity recommendations.

The present study has several limitations. Although the accelerometer has been considered a gold standard method to measure physical activities in free-living conditions, it was not possible to measure the type and the context in which the physical activity was performed, which hinders the analysis of what kind of activities were most often performed by these patients. In addition, the accelerometer does not assess physical activities such as water gymnastics and resistance training, which are commonly performed by elderly patients, and could underestimate the real physical activity levels of our sample. Given that there are no specific physical activity recommendation 
for PAD patients, we employed the current physical activity recommendations for the overall population. However, whether this approach is ideal for PAD patients is unknown. The study was performed in São Paulo, Brazil, and our results may not be extrapolated to other patients with different cultures and lifestyle. We did not include a matched overall population group to compare the prevalence of physical activity between nonPAD and PAD patients. Finally, we did not analyze the type of physical activity performed by these patients, or the difference in physical activities over the year. Some patients assessed during colder/rainier months could be less active than those assessed in the summer months.

\section{Conclusion}

This study showed that the pattern of physical activity of Brazilian PAD patients with intermittent claudication symptoms are characterized by a high amount of time spent in sedentary behavior and a low engagement in MVPA, with only $3.4 \%$ of these patients meeting the current physical activity recommendations for the overall population. Moreover, younger patients, regardless of clinical and functional factors, are more likely to meet the current physical activity recommendations.

\section{Author contributions}

Conception and design of the research: Zeratti $\mathrm{AE}$, Puech-Leão P, Wolosker N, Ritti-Dias RM, Cucato GG; Acquisition of data: Correia MA, Oliveira PML, Palmeira AC,
Domingues WJR; Analysis and interpretation of the data: Gerage AM; Statistical analysis: Gerage AM, Correia MA, Ritti-Dias RM, Cucato GG; Writing of the manuscript: Gerage AM, Correia MA, Oliveira PML, Palmeira AC, Domingues WJR, Ritti-Dias RM; Critical revision of the manuscript for intellectual content: Zeratti AE, Puech-Leão P, Wolosker N, Cucato GG.

\section{Potential Conflict of Interest}

No potential conflict of interest relevant to this article was reported.

\section{Sources of Funding}

This study was funded by CNPq-409707/2016-3. Coordenação de aperfeiçoamento do ensino superior - CAPES

\section{Study Association}

This study is not associated with any thesis or dissertation work.

\section{Ethics approval and consent to participate}

This study was approved by the Ethics Committee of the Hospital Israelita Albert Einstein under the protocol number CAAE: 42379015.3.0000.0071. All the procedures in this study were in accordance with the 1975 Helsinki Declaration, updated in 2013. Informed consent was obtained from all participants included in the study.

\section{References}

1. Fowkes FG, Rudan D, Rudan I, Aboyans V, Denenberg JO, McDermott $\mathrm{MM}$, et al. Comparison of global estimates of prevalence and risk factors for peripheral artery disease in 2000 and 2010: a systematic review and analysis. Lancet. 2013;382(9901):1329-40.

2. Farah BQ, Ritti-Dias RM, Cucato GG, Chehuen Mda R, Barbosa JP, Zeratti $A E$, et al. Effects of clustered comorbid conditions on walking capacity in patients with peripheral artery disease. Ann Vasc Surg. 2014;28(2):279-83.

3. Gerhard-Herman MD, Gornik HL, Barrett C, Barshes NR, Corriere MA, Drachman DE, et al. 2016 AHA/ACC Guideline on the Management of Patients With Lower Extremity Peripheral Artery Disease: Executive Summary: A Report of the American College of Cardiology/American Heart Association Task Force on Clinical Practice Guidelines. J Am Coll Cardiol. 2017;69(11):1465-508.

4. Cavalcante BR, Ritti-Dias RM, Soares AH, Lima AH, Correia MA, De Matos LD, et al. A single bout of Arm-crank exercise promotes positive emotions and post-exercise hypotension in patients with symptomatic peripheral artery disease. Eur J Vasc Endovasc Surg. 2017;53(2):223-8.

5. Chehuen M, Cucato GG, Carvalho CRF, Ritti-Dias RM, Wolosker N, Leicht AS, et al. Walking training at the heart rate of pain threshold improves cardiovascular function and autonomic regulation in intermittent claudication: a randomized controlled trial. J Sci Med Sport. 2017;20(10):886-92.

6. Ritti-Dias RM, Wolosker N, de Moraes Forjaz CL, Carvalho CR, Cucato GG, Leao PP, et al. Strength training increases walking tolerance in intermittent claudication patients: randomized trial. J Vasc Surg. 2010;51(1):89-95.

7. Gardner AW. Exercise rehabilitation for peripheral artery disease: An exercise physiology perspective with special emphasis on the emerging trend of home-based exercise. VASA. 2015;44(6):405-17.

8. World Health Organization. Global Recommendations on Physical Activity for Health. Geneva; 2010.

9. Barbosa JP, Farah BQ, Chehuen M, Cucato GG, Farias Junior JC, Wolosker N, et al. Barriers to physical activity in patients with intermittent claudication. Int J Behav Med. 2015;22(1):70-6.

10. Aboyans V, Criqui MH, Abraham P, Allison MA, Creager MA, Diehm C, et al. Measurement and interpretation of the ankle-brachial index: a scientific statement from the American Heart Association. Circulation. 2012;126(24):2890-909.

11. Cavalcante BR, Ritti-Dias RM, Germano Soares AH, Domingues WJR, Saes GF, Duarte FH, et al. Graduated compression stockings does not decrease walking capacity and muscle oxygen saturation during 6-minute walk test in intermittent claudication patients. Ann Vasc Surg. 2017 Apr;40:239-42.

12. Ritti-Dias RM, Gobbo LA, Cucato GG, Wolosker N, Jacob Filho W, Santarem JM, et al. Translation and validation of the walking impairment questionnaire in Brazilian subjects with intermittent claudication. Arq Bras Cardiol. 2009;92(2):136-49.

13. Cucato GG, Correia MA, Farah BQ, Saes GF, Lima AH, Ritti-Dias RM, et al. Validation of a Brazilian Portuguese Version of the Walking Estimated-Limitation Calculated by History (WELCH). Arq Bras Cardiol. 2016;106(1):49-55.

14. Copeland JL, Esliger DW. Accelerometer assessment of physical activity in active, healthy older adults. J Aging Phys Act. 2009;17(1):17-30. 
15. Buman MP, Hekler EB, Haskell WL, Pruitt L, Conway TL, Cain KL, et al. Objective light-intensity physical activity associations with rated health in older adults. Am J Epidemiol. 2010;172(10):1155-65.

16. Evenson KR, Butler EN, Rosamond WD. Prevalence of physical activity and sedentary behavior among adults with cardiovascular disease in the United States. J Cardiopulm Rehabil Prev. 2014;34(6):406-19.

17. Butler EN, Evenson KR. Prevalence of physical activity and sedentary behavior among stroke survivors in the United States. Top Stroke Rehabil. 2014;21(3):246-55

18. Tucker JM, Welk GJ, Beyler NK. Physical activity in U.S.: adults compliance with the Physical Activity Guidelines for Americans. Am J Prev Med. 2011;40(4):454-61.

19. Ramires VV, Wehrmeister FC, Bohm AW, Galliano L, Ekelund U, Brage S, et al. Physical activity levels objectively measured among older adults: a population-based study in a Southern city of Brazil. Int J Behav Nutr Phys Act. 2017;14(1):13.

20. Dunlop DD, Song J, Semanik PA, Chang RW, Sharma L, Bathon JM, et al Objective physical activity measurement in the osteoarthritis initiative: are guidelines being met? Arthritis Rheum. 2011;63(11):3372-82.

21. Kao MC, Jarosz R, Goldin M, Patel A, Smuck M. Determinants of physical activity in America: a first characterization of physical activity profile using the National Health and Nutrition Examination Survey (NHANES). PM R. 2014;6(10):882-92.

22. Brevetti G, Martone VD, Perna S, Cacciatore F, Corrado S, Di Donato A, et al. Intermittent claudication and risk of cardiovascular events. Angiology. 1998;49(10):843-8.

23. Grenon SM, Chong K, Alley H, Nosova E, Gasper W, Hiramoto J, et al. Walking disability in patients with peripheral artery disease is associated with arterial endothelial function. J Vasc Surg. 2014;59(4):1025-34.

24. Gardner AW, Parker DE, Montgomery PS, Sosnowska D, Casanegra AI, Ungvari Z, et al. Endothelial cell inflammation and antioxidant capacity are associated with exercise performance and microcirculation in patients with symptomatic peripheral artery disease. Angiology. 2015;66(9):867-74.

25. Farah BQ, Souza Barbosa JP, Cucato GG, Chehuen Mda R, Gobbo LA, Wolosker $\mathrm{N}$, et al. Predictors of walking capacity in peripheral arterial disease patients. Clinics (Sao Paulo). 2013;68(4):537-41.

26. Gardner AW, Ritti Dias RM, Khurana A, Parker DE. Daily ambulatory activity monitoring in patients with peripheral artery disease. Phys Ther Rev. 2010;15(3):212-23

27. Gommans LN, Hageman D, Jansen I, de Gee R, van Lummel RC, Verhofstad $\mathrm{N}$, et al. Minimal correlation between physical exercise capacity and daily activity in patients with intermittent claudication. J Vasc Surg. 2016;63(4):983-9.

28. Gerage AM, Benedetti TR, Farah BQ, Santana Fda S, Ohara D, Andersen $\mathrm{LB}$, et al. Sedentary behavior and light physical activity are associated with brachial and central blood pressure in hypertensive patients. PLoS One. 2015;10(12):e0146078.

29. Germano-Soares AH, Andrade-Lima A, Meneses AL, Correia MA, Parmenter BJ, Tassitano RM, et al. Association of time spent in physical activities and sedentary behaviors with carotid-femoral pulse wave velocity: A systematic review and meta-analysis. Atherosclerosis. 2018 Feb;269:211-8.

30. Healy GN, Wijndaele K, Dunstan DW, Shaw JE, Salmon J, Zimmet PZ, et al Objectively measured sedentary time, physical activity, and metabolic risk: the Australian Diabetes, Obesity and Lifestyle Study (AusDiab). Diabetes Care. 2008;31(2):369-71

31. Kim J, Tanabe K, Yokoyama N, Zempo H, Kuno S. Objectively measured lightintensity lifestyle activity and sedentary time are independently associated with metabolic syndrome: a cross-sectional study of Japanese adults. Int J Behav Nutr Phys Act. 2013 Mar 4;10:30.

32. Garg PK, Tian L, Criqui MH, Liu K, Ferrucci L, Guralnik JM, et al. Physical activity during daily life and mortality in patients with peripheral arterial disease. Circulation. 2006;114(3):242-8. 